\title{
Consumption of Repeatedly Heated Soy Oil Increases the Serum Parameters Related to Atherosclerosis in Ovariectomized Rats
}

\author{
Siti Khadijah Adam, ${ }^{1}$ Srijit Das, ${ }^{2}$ Ima Nirwana Soelaiman, ${ }^{1}$ Nor Aini Umar ${ }^{3}$ \\ and KAMSIAH JAARIN ${ }^{1}$ \\ ${ }^{1}$ Department of Pharmacology, Faculty of Medicine, University Kebangsaan Malaysia, Kuala Lumpur, \\ Malaysia \\ ${ }^{2}$ Department of Anatomy, Faculty of Medicine, University Kebangsaan Malaysia, Kuala Lumpur, \\ Malaysia \\ ${ }^{3}$ Department of Pathology, Faculty of Medicine, University Kebangsaan Malaysia, Kuala Lumpur, \\ Malaysia
}

\begin{abstract}
Repeated heating of soy oil may promote lipid peroxidation. Oxidized unsaturated fatty acids may contribute to the pathogenesis of atherosclerosis, especially in estrogen-deficient states. This study was performed to explore the deleterious effects of repeatedly heated soy oil on the development of atherosclerosis using ovariectomized rats, which represent an estrogen-deficient state. Twenty-four female Sprague-Dawley rats were ovariectomized and were divided equally into four groups. The control group was fed with $2 \%$ cholesterol diet without any oil. The three treatment groups each received $2 \%$ cholesterol diet fortified with fresh, once-heated or five-times-heated (repeatedly heated) soy oil, respectively. Serum thiobarbituric acid reactive substances (TBARS), lipid profile and homocysteine levels were measured prior to ovariectomy and at the end of four months. Ovariectomized rats treated with repeatedly heated soy oil showed significant increases in lipid peroxidation and low-density lipoprotein (LDL) levels. Treatment with once-heated or repeatedly heated soy oil caused a significant increase in total cholesterol, while fresh soy oil caused significant reduction in homocysteine level as compared to other groups. Repeatedly heated soy oil caused significant increases in TBARS and LDL as compared to fresh oil. The higher level of homocysteine in the ovariectomized rats fed with repeatedly heated oil, as compared to those fed with fresh oil, also suggests the repeatedly heated oil contributes to the development of atherosclerosis. Importantly, the protective effect of the soy oil may be lost once it was being repeatedly heated. In conclusion, the consumption of repeatedly heated oil may predispose to atherosclerosis in estrogen-deficient states. soy oil; heating; ovariectomy; atherosclerosis; lipid peroxidation; free radicals.
\end{abstract}

Tohoku J. Exp. Med., 2008, 215 (3), 219-226.

(C) 2008 Tohoku University Medical Press

Received March 25, 2008; revision accepted for publication May 26, 2008.

Correspondence: Prof. Dr. Kamsiah Jaarin, M.D., M.Sc., Department of Pharmacology, Faculty of Medicine, University Kebangsaan Malaysia, Jalan Raja Muda Abd. Aziz, 50300 Kuala Lumpur, Malaysia.

e-mail:kamsiah@medic.ukm.my 
Atherosclerotic lesions in human and animals are related to elevated levels of total cholesterol and low-density lipoprotein (LDL). The incidence of atherosclerosis in women is lower as compared to men in the same age group but its incidence increases after menopause due to decrease in estrogen level (Rossouw 2002). The above fact is being best explained by the fact that estrogen has cardio-protective effects which can reduce the LDL level (Nabulsi et al. 1993) and has antioxidant action against lipid peroxidation (Badeau et al. 2005). Lipid deposition on the arterial wall likely begins with oxidation of LDL in the vessel wall (Osterud and Bjorklid 2003). Oxidized LDL (oLDL) may act as chemotactic agent attracting monocytes and macrophages to the endothelium which then develops into lipid laden foam cells of an atheromatous plaque (Plutzky and Libby 2003).

During frying, cooking oil is often exposed to high temperatures for a long period of time. This practice generates lipid peroxidation products that may be harmful for human health (Lapointe et al. 2006). Our population is prone to using the same oil for repeated frying in order to cut down the cost of cooking but they may not be aware of the fact that such practice may generate more lipid peroxidation products which are harmful to health.

Several studies have demonstrated the adverse effects of oxidized dietary fats on human and experimental animals; namely, consumption of oxidized oil caused liver dysfunction (Hayam et al. 1995; Owu et al. 1998) and was responsible for development of fatty streaks (Staprans et al. 1996; Hur et al. 2005).

Considering the potential hazardous effect of oxidized oil, we embarked on this study to determine the harmful effects of repeatedly heated soy oil consumed along with $2 \%$ cholesterol diet. The ovariectomized rats resembled an estrogen-deficient state of post-menopausal period and the risk factors related to atherosclerosis were found to increase in such. The results of our study provide valuable information on the harmful effects of repeated heating of the same cooking oil which may be dangerous for the estrogen deficient state of the post-menopausal period.

\section{Methods}

\section{Experimental animals}

Twenty-four healthy and mature female SpragueDawley rats (200-250 g) were obtained from the Animal Unit, University Kebangsaan Malaysia. The rats were kept in stainless steel cages at room temperature $(27 \pm$ $2{ }^{\circ} \mathrm{C}$ ), under natural 12-hr light cycle. All rats had free access to water and were administered $150 \mathrm{~g}$ of test diet/ week. This study was approved by the University Kebangsaan Malaysia Animal Ethic Committee (UKM AEC: FAR/2003/ Kamsiah/25 Jan/ 090).

\section{Source and preparation of diets}

Soy oil (Yee Lee Edible Oils, Yee Lee Corporation Bhd, Perak, Malaysia) in three forms i.e., fresh, heated once or heated five times (as described by Owu et al. 1998) were used. The heating process involved using 2.5 $\mathrm{L}$ of the soy oil to fry $1 \mathrm{~kg}$ (approximately 25 pieces) of "keropok lekor" (fish-flavored chips) in a metal wok. The temperature of the heated oil reached about $180^{\circ} \mathrm{C}$, and the cooking process lasted for about $10 \mathrm{~min}$. While heating the oil 5 times, it was cooled for $5 \mathrm{hrs}$ in between heating, then the whole frying processes was repeated with a fresh batch of "keropok lekor". No fresh oil was added between batches to make up for the loss due to uptake of the oil by the frying material.

Two percent cholesterol diet was obtained from MP Biomedicals, Inc. (Seven Hills, Australia). The test diets were formulated by mixing $15 \%(\mathrm{w} / \mathrm{w})$ of soy oil with ground $2 \%$ cholesterol diet. The pellets were reformed and dried in an oven at $80^{\circ} \mathrm{C}$ overnight.

\section{Study design}

The rats $(n=24)$ were allowed to acclimatize for 1 week, prior to treatment and were ovariectomized ahead of the study. They were randomly divided equally into four groups:- one group was fed with $2 \%$ cholesterol diet alone (control) while another three groups were fed with $2 \%$ cholesterol diet fortified with fresh soy oil (FSO), heated once soy oil (1HSO) and heated 5 times soy oil (5HSO), respectively for 4 months. The mean body weight and food intake were taken weekly during the study period. The 18 -hr fasting serum were taken prior to ovariectomy and at the end of 4 months of study. Serum was stored at $-70^{\circ} \mathrm{C}$ for further analyses. 
Serum thiobarbituric acid reactive substances (TBARS)

TBARS level in the serum was determined using a method described by Ledwozyw et al. (1986) with some modifications. An amount of $0.5 \mathrm{ml}$ sample was acidified with $2.5 \mathrm{ml}$ of $1.22 \mathrm{M}$ trichloroacetic acid (TCA)/0.6 $\mathrm{M}$ hydrochloric acid $(\mathrm{HCl})$ and left to stand at room temperature for $15 \mathrm{~min}$. Then, $1.5 \mathrm{ml}$ of $0.67 \%$ thiobarbituric acid (TBA)/0.05 $\mathrm{M}$ sodium hydroxide $(\mathrm{NaOH})$ was added. The samples were incubated in a $100^{\circ} \mathrm{C}$ water bath for $30 \mathrm{~min}$. Subsequently, they were left to cool at room temperature before the addition of $4 \mathrm{ml}$ of n-buthanol. After thorough mixing, the mixture was centrifuged for $10 \mathrm{~min}$ at 3,000 rpm. The absorbency of the upper phase was read at Ex: 515 Em: 553 by using spectrofluorometer (Shimadzu RF500, Kyoto, Japan).

The protein content in the serum was determined using a method described by Lowry et al. (1951) with some modifications. $0.5 \mathrm{ml}$ sample was added with $5 \mathrm{ml}$ mixture of $2 \%$ sodium carbonate $\left(\mathrm{Na}_{2} \mathrm{CO}_{3}\right), 2 \%$ sodium or potassium tartrate $(\mathrm{Na} / \mathrm{K}$ tartrate) and $1 \%$ copper sulphate solution $\left(\mathrm{CuSO}_{4} .5 \mathrm{H}_{2} \mathrm{O}\right)$ with ratio $100: 1: 1$. Subsequently, they were left to cool at room temperature for $15 \mathrm{~min}$ before the addition of $0.5 \mathrm{ml}$ of diluted FolinCiocalteau phenol reagent. After $35 \mathrm{~min}$, the optical density was measured at $70 \mathrm{~nm}$ with spectrophotometer (Shimadzu UV-160A, Japan). The serum TBARS was calculated as serum TBARS/protein.

\section{Serum lipid profile}

Serum lipid profile was determined enzymatically using commercially available kits from Randox Laboratories Ltd. (Crumlin, Co. Antrim, UK). These tests were performed using Vitalab Selectra E (Vital Scientific, Dieren, Netherlands) following the manufacturer's instructions.

\section{Serum homocysteine}

Serum homocysteine level was determined by Fluorescence Polarization Immunoassay (FPIA) using commercially available kits from Abbott Laboratories (Illinois, USA). The test was performed using Cobas Integra (Roche Professional Diagnostics, Basel, Switzerland) following the manufacturer's instructions.

\section{Statistical analysis}

The data was presented as the mean percentage \pm standard error of mean (S.E.M.). The normality of all the data was first determined by using KolmogorovSmirnorv test. Paired $t$-test was used to analyze level differences between before ovariectomy and after four months of treatment data. To analyse TBARS, lipid profiles and homocysteine levels among the experimental groups, analysis of variance (ANOVA) followed by Tukey hsd post-hoc test was used if the data was normal. Data which were not normally distributed were analyzed using non-parametric tests, i.e., Kruskal-Wallis $\mathrm{H}$ and Mann-Whitney's U-tests. A value of $p<0.05$ was considered significant. All analyses were conducted using Statistical Product and Service Solutions (SPSS) 11.5.0 software (Chicago, IL, USA).

\section{Results}

All the parameters were measured before ovariectomy and after 4 months of treatment in the experimental rats and tabulated in Table 1.

\section{Food intake and body weight}

There was no significance difference in food intake among the oil fed groups at the end of study. All treatment groups had less food intake compared to control. Rats treated with fresh and once-heated soy oil showed lower increase in body weight compared to control and 5HSO.

\section{Serum TBARS}

The percentage increase in serum TBARS was significantly higher in the $1 \mathrm{HSO}$ and $5 \mathrm{HSO}$ as compared to control and FSO. The 5HSO group which showed more than $100 \%$ of increase which was significantly higher compared to $1 \mathrm{HSO}$.

\section{Serum total cholesterol (TC)}

There was an increasing trend in the serum $\mathrm{TC}$ in all the treatment groups (Fig. 1). All treatment groups showed more than $100 \%$ increase of TC. The percentage increase in serum TC was significantly higher in $1 \mathrm{HSO}$ and $5 \mathrm{HSO}$ compared to control and FSO.

\section{Serum $L D L$}

There was also an increasing trend in serum LDL in all the treatment groups (Fig. 2). All groups, including control showed more than $100 \%$ increase of LDL. The percentage increase in serum LDL was significantly the highest in 
TABLE 1. Various parameters measured before ovariectomy and after 4 months of treatment.

\begin{tabular}{|c|c|c|c|}
\hline & Group & Before ovariectomy & After 4 months of treatment \\
\hline \multirow[t]{4}{*}{ TBARS (nmol/mg protein) } & Control & $0.077 \pm 0.004$ & $0.105 \pm 0.012 *$ \\
\hline & FSO & $0.070 \pm 0.003$ & $0.080 \pm 0.002 *$ \\
\hline & $1 \mathrm{HSO}$ & $0.076 \pm 0.002$ & $0.130 \pm 0.011 *$ \\
\hline & $5 \mathrm{HSO}$ & $0.081 \pm 0.004$ & $0.171 \pm 0.006 *$ \\
\hline \multirow[t]{4}{*}{$\mathrm{TC}(\mathrm{mmol} / \mathrm{L})$} & Control & $1.82 \pm 0.09$ & $2.86 \pm 0.24 *$ \\
\hline & FSO & $1.42 \pm 0.09$ & $2.92 \pm 0.40 *$ \\
\hline & $1 \mathrm{HSO}$ & $1.28 \pm 0.11$ & $4.61 \pm 0.52 *$ \\
\hline & $5 \mathrm{HSO}$ & $1.25 \pm 0.13$ & $5.96 \pm 0.18 *$ \\
\hline \multirow[t]{4}{*}{$\mathrm{LDL}(\mathrm{mmol} / \mathrm{L})$} & Control & $0.33 \pm 0.02$ & $0.69 \pm 0.02 *$ \\
\hline & FSO & $0.23 \pm 0.01$ & $0.64 \pm 0.02 *$ \\
\hline & $1 \mathrm{HSO}$ & $0.26 \pm 0.02$ & $0.96 \pm 0.16 *$ \\
\hline & $5 \mathrm{HSO}$ & $0.21 \pm 0.02$ & $1.17 \pm 0.22 *$ \\
\hline \multirow[t]{4}{*}{$\mathrm{TG}(\mathrm{mmol} / \mathrm{L})$} & Control & $0.49 \pm 0.05$ & $0.61 \pm 0.05$ \\
\hline & FSO & $0.39 \pm 0.02$ & $0.49 \pm 0.04 *$ \\
\hline & $1 \mathrm{HSO}$ & $0.37 \pm 0.03$ & $0.50 \pm 0.03 *$ \\
\hline & $5 \mathrm{HSO}$ & $0.38 \pm 0.01$ & $0.53 \pm 0.03 *$ \\
\hline \multirow[t]{4}{*}{$\mathrm{HDL}(\mathrm{mmol} / \mathrm{L})$} & Control & $0.64 \pm 0.01$ & $0.47 \pm 0.04 *$ \\
\hline & FSO & $0.51 \pm 0.02$ & $0.43 \pm 0.02 *$ \\
\hline & $1 \mathrm{HSO}$ & $0.52 \pm 0.02$ & $0.36 \pm 0.04 *$ \\
\hline & $5 \mathrm{HSO}$ & $0.49 \pm 0.03$ & $0.24 \pm 0.03 *$ \\
\hline \multirow[t]{4}{*}{ Homocysteine $(\mu \mathrm{mol} / \mathrm{L})$} & Control & $4.30 \pm 0.28$ & $6.50 \pm 0.40 *$ \\
\hline & FSO & $6.57 \pm 0.46$ & $7.39 \pm 0.43$ \\
\hline & $1 \mathrm{HSO}$ & $6.01 \pm 0.60$ & $7.86 \pm 0.41 *$ \\
\hline & $5 \mathrm{HSO}$ & $5.64 \pm 0.46$ & $7.80 \pm 0.44 *$ \\
\hline
\end{tabular}

Data are shown as mean \pm S.E.M. $(n=6)$.

* significant difference between before ovariectomy and after 4 months of treatment $(p<0.05)$.

TBARS, thiobarbituric acid reactive substances; TC, total cholesterol; LDL, low-density lipoprotein; TG, triglyceride; HDL, high-density lipoprotein; FSO, fresh soy oil treated group; 1HSO, once-heated soy oil treated group; 5HSO, five-times-heated soy oil treated group.

5HSO compared to control and other treatment groups.

\section{Serum triglyceride}

All groups showed an increase in serum triglyceride after treatment. However, there was no significant difference in serum triglyceride concentration amongst the groups.

Serum high-density lipoprotein (HDL)

All groups showed a decrease in serum HDL after treatment. Similar to triglyceride, there was no significant difference in serum HDL concentration amongst the groups. 


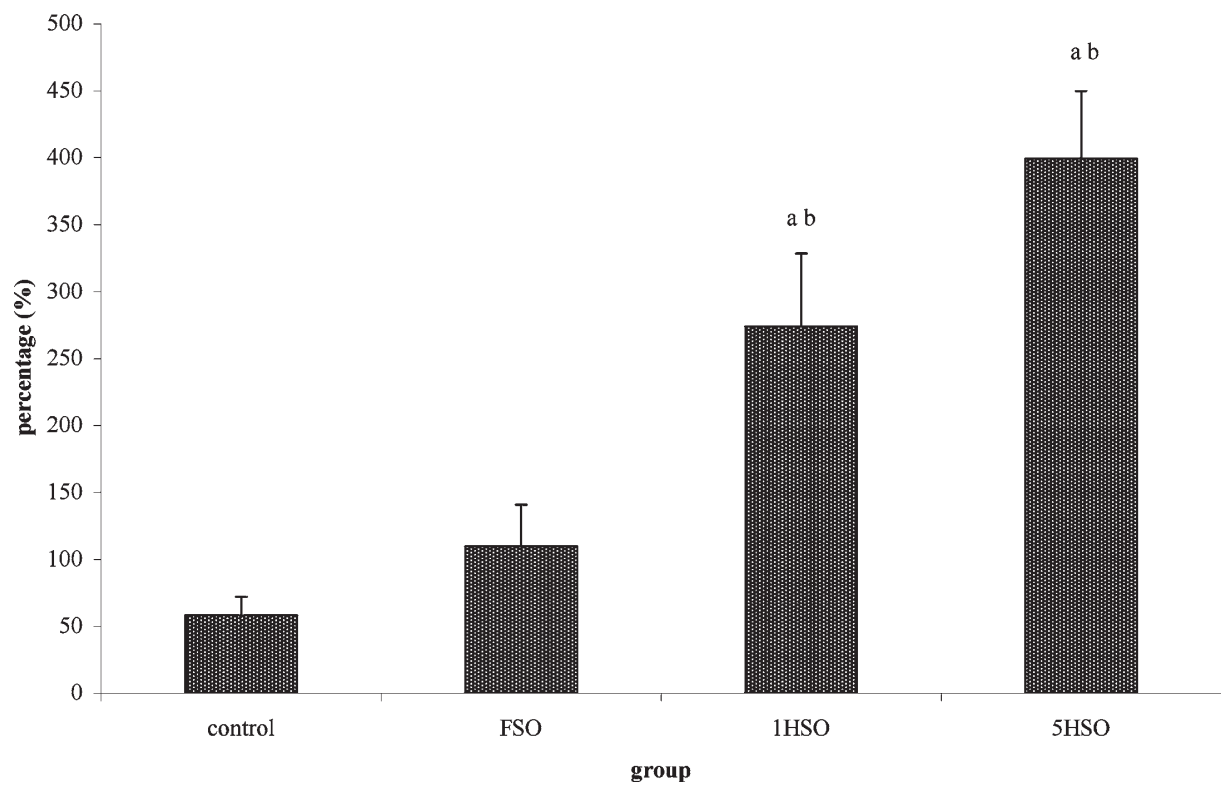

Fig. 1. Percentage changes in serum TC with fresh and heated soy oil after 4 months of feeding. Error bars represent the S.E.M.

a, significant compared to control $(p<0.05)$.

$\mathrm{b}$, significant compared to FSO $(p<0.05)$.

FSO, fresh soy oil treated group; $1 \mathrm{HSO}$, once-heated soy oil treated group; $5 \mathrm{HSO}$, five-times-heated soy oil treated group.

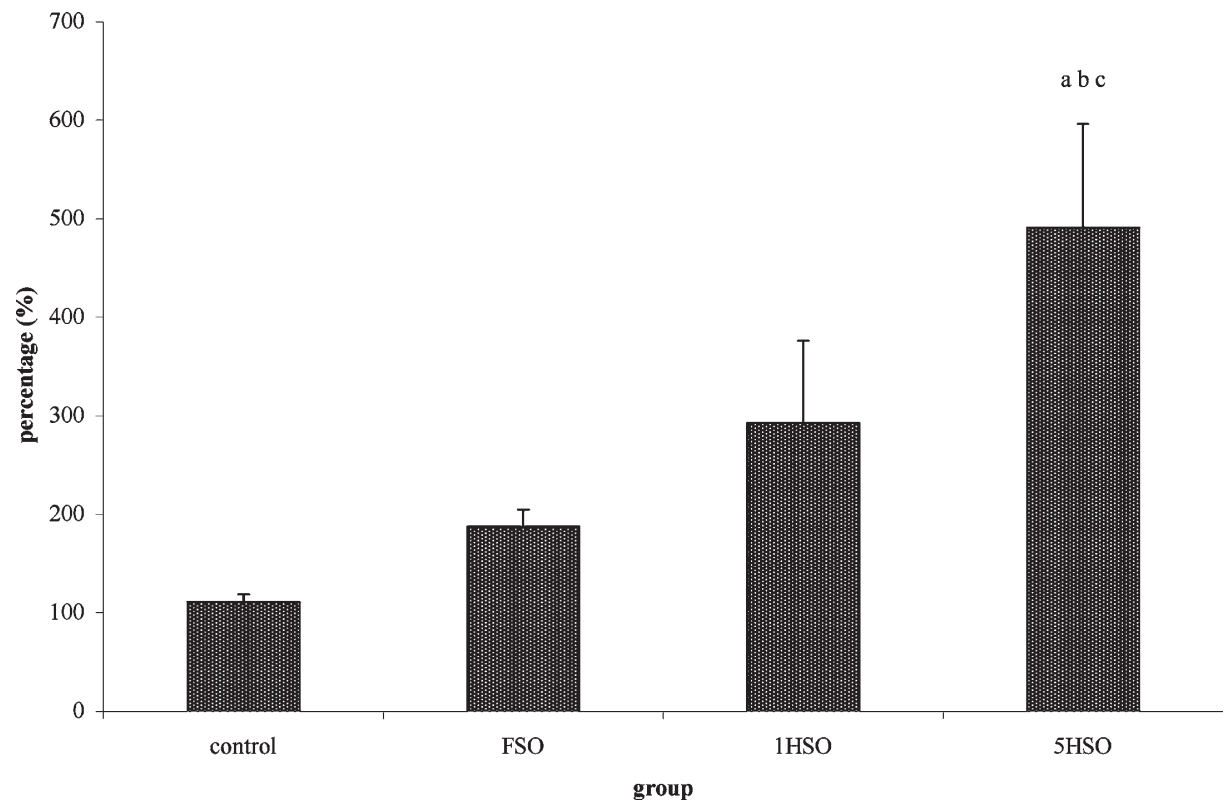

Fig. 2. Percentage changes in serum LDL with fresh and heated soy oil after 4 months of feeding. Error bars represent the S.E.M.

a, significant compared to control $(p<0.05)$.

$\mathrm{b}$, significant compared to FSO $(p<0.05)$.

c, significant compared to $1 \mathrm{HSO}(p<0.05)$.

FSO, fresh soy oil treated group; $1 \mathrm{HSO}$, once-heated soy oil treated group; $5 \mathrm{HSO}$, five-times-heated soy oil treated group. 


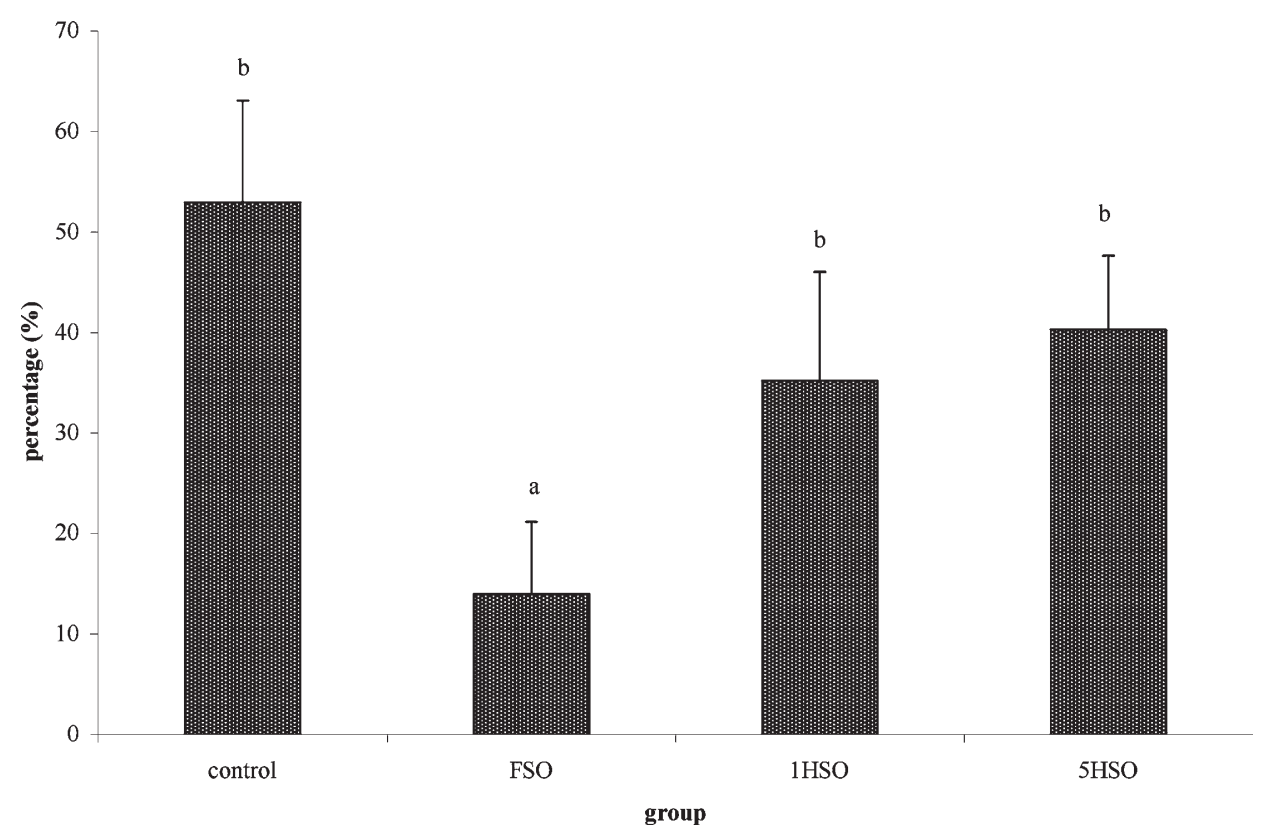

Fig. 3. Percentage changes in serum homocysteine with fresh and heated soy oil after 4 months of feeding. Error bars represent the s.E.M.

a, significant compared to control $(p<0.05)$.

$\mathrm{b}$, significant compared to FSO $(p<0.05)$.

FSO, fresh soy oil treated group; 1HSO, once-heated soy oil treated group; 5HSO, five-times-heated soy oil treated group.

\section{Serum homocysteine}

There was a significant difference in serum homocysteine level of FSO as compared to control and other treatment groups (Fig. 3). The FSO group showed a significantly lower homocysteine level as compared to other groups.

\section{DiscuSSION}

We conducted this study in ovariectomized rats, which may simulate a post-menopausal condition characterized by the absence of ovarian hormones, like estrogen. The effects of repeatedly heated soy oil on lipid peroxidation, lipid profile and the homocysteine level were studied in detail. Interestingly, these parameters are related to cardiovascular diseases, particularly atherosclerosis. We propose that thermally oxidized soy oil generates free radicals which enhance the oxidative stress secondary to an estrogen-deficient state and cholesterol laden diet.

Our study showed a significant reduction in food intake when their diet was fortified with soy oil, either fresh or heated compared to control which received the $2 \%$ cholesterol diet alone. This might be due to the addition of the oil that modify the taste and smell of their diet. However, heating process which caused physical changes in the oil did not have any significant effects on their food intake. This was proven by the similar food intake amongst the treatment groups. In addition to the low food intake, FSO and 1HSO showed lower body weight gain compared to control and 5HSO. On the other hand, 5HSO showed a significant increase in the body weight despite their low food intake. The reason for this is not well understood, but their weight gain may be due to increased fat deposition in adipose tissue, which might contribute to the weight increase.

We determined the level of lipid peroxidation by measuring serum TBARS. There is a possibility that the increase of TBARS in the control (about 24\%) resulted due to estrogen deficiency caused by ovariectomy. This estrogen-deficient state may exactly simulate a post-menopausal scenario. However, the $1 \mathrm{HSO}$ and $5 \mathrm{HSO}$ had a larger percentage of increase, which was signifi- 
cant as compared to the control and FSO. Moreover, 5HSO showed the highest percentage of TBARS increase as compared to other groups. This proves that repeated heating may generate more free radicals, as reported by Nwanguma et al. (1999). Soy oil, which contains high polyunsaturated fatty acids (Cottrell 1991) is more susceptible to oxidation (Rueda-Clausen et al. 2007). Thus, it may explain the discrepancies in the results compared to our previous work which had used a different type of cooking oil (Kamsiah et al. 2006).

We had observed in our previous work that heating caused reduction of vitamin $\mathrm{E}$ in soy oil (Adam et al. 2007). It thus appears that the increase of lipid peroxidation in this present study was inversely proportional to the amount of vitamin E contained in the respective oils. This proved that the major biological role of vitamin $\mathrm{E}$ as an antioxidant was gradually lost while being heated repeatedly.

There was an increase in serum TC in all the groups. The increase in these groups was secondary to $2 \%$ cholesterol diet intake. Intake of heated soy oil, both once and repeatedly heated, accelerated the increase in serum $\mathrm{TC}$ in the rats. This finding was contrary to the earlier study by Hur et al. (2005) which observed that heated corn oil reduced plasma cholesterol in rabbits. We are not sure about the reason for this inconsistency but obviously the type of animal, type of oil and ovariectomized state might be the contributing factors.

Serum LDL also showed an increasing trend similar to TC. The 5HSO group showed the highest LDL increase as compared to other groups. This indicates that the increase of LDL level was directly related to the repeated heating. Therefore, the progression of atherosclerosis may worsen when repeatedly heated oil is being consumed and subsequently it causes an increase in the LDL level in the blood. The increase will enhance the oxidation of LDL in vivo by certain pathways that promote atherogenesis (Heinecke 1998). In fact, dietary guidelines have been laid down to check the secondary prevention of cardiovascular diseases (Mead et al. 2006) and the innocent popula- tion need to be warned of such harmful effects. One can imagine the harmful effects which would be caused if the heated soy oil is consumed by post-menopausal women who already have an existing estrogen deficiency state.

Both serum triglyceride and HDL showed no significant difference between the groups. Our result on triglyceride was contradictory to the findings of Rueda-Clausen et al. (2007) which reported that consumption of deep-fried palm oil caused an acute increase of serum triglyceride level in humans. The differences in preparation of the oil, subject's metabolic conditions and duration of the study are the possible factors that might have contributed to these discrepancies. HDL was found to be decreased in all the animals and this might related to the estrogen deficiency. No differences amongst the treatment groups indicated that the degree of heating did not interfere with the changes in HDL level.

We also measured the homocysteine level which is known to be an independent risk factor in atherosclerosis (Su et al. 2005). We found that the level of homocysteine increased markedly after ovariectomy. Feeding with fresh soy oil may not be harmful as compared to the ingestion of heated oil. There is a possibility that the destruction of the vitamin $\mathrm{E}$ in the oil may contribute to the harmful effect.

\section{Conclusion}

Our study has shown that repeated heating of soy oil might cause an increase in lipid peroxidation and LDL in an ovariectomized model of rat which simulates a post-menopausal state with estrogen deficiency. From these results, it was obvious that repeated heating gradually diminished the protective effects of soy oil and may contribute to the pathogenesis of atherosclerosis especially in post-menopausal women. We suggest that the common practice of using repeatedly heated soy oil be discontinued in general population in order to avoid the harmful effects.

\section{Acknowledgments}

This study was funded by grant IRPA 06-02-02-0050-EA242. The authors wish to thank 
Puan Azizah Othman, Sinar Suriya Muhamad as well as other staff members in the Chemical Pathology Laboratory, HUKM for valuable assistance.

\section{References}

Adam, S.K., Sulaiman, N.A., Md Top, A.G. \& Jaarin, K. (2007) Heating reduces vitamin E content in palm and soy oils. Malays. J. Biochem. Molec. Biol., 15, 76-79.

Badeau, M., Adlercreutz, H., Kaihovaara, P. \& Tikkanen, M.J. (2005) Estrogen A-ring structure and antioxidative effect on lipoproteins. J. Steroid Biochem. Mol. Biol., 96, 271278.

Cottrell, R.C. (1991) Introduction: Nutritional aspects of palm oil. Am. J. Clin. Nutr., 53, 989S-1009S.

Hayam, I., Cogan, U. \& Mokady, S. (1995) Dietary oxidized oil and the activity of antioxidant enzymes and lipoprotein peroxidation in rats. Nutr. Res., 15, 1037-1044.

Heinecke, J.W. (1998) Oxidants and antioxidants in the pathogenesis of atherosclerosis: implications for the oxidized low density lipoprotein hypothesis. Atherosclerosis, 141, $1-15$.

Hur, S.J., Du, M., Williamson, M. \& Ahn, D.U. (2005) Effect of dietary fats on blood cholesterol and lipid and the development of atherosclerosis in rabbits. Nutr. Res., 25, 925-935.

Kamsiah, J., Norhayati, M., Norzana, G., Nor Aini, U. \& Ima Nirwana, S. (2006) Effects of heated vegetable oils on serum lipids and aorta of ovariectomized rats. Pak. J. Nutr., 5, 19-29.

Lapointe, A., Couillard, C. \& Lemieux, S. (2006) Effects of dietary factors on oxidation of low-density lipoprotein particles. J. Nutr. Biochem., 17, 645-658.

Ledwozyw, A., Michalak, J., Stepien, A. \& Kadziolka, A. (1986) The relationship between plasma triglycerides, cholesterol, total lipids and lipid peroxidation products during human atherosclerosis. Clin. Chim. Acta, 155, 275-283.

Lowry, O.H., Rosebrugh, N.J., Farr, A.L. \& Randall, R.J. (1951) Protein measurement with the Folin phenol reagent. $J$. Biol. Chem., 193, 265-275.

Mead, A., Atkinson, G., Albin, D., Alphey, D., Baic, S., Boyd, O., Cadigan, L., Clutton, L., Craig, L., Flanagan, C., Greene, P., Griffiths, E., Lee, N.J., Li, M., McKechnie, L., Ottaway, J., Paterson, K., Perrin, L., Rigby, P., Stone, D., Vine, R., Whitehead, J., Wray, L., Hooper, L. on behalf of the UK
Heart Health and Thoracic Dietitians Interest Group (Specialist group of the British Dietetic Association) (2006) Dietetic guidelines on food and nutrition in the secondary prevention of cardiovascular disease - evidence from systematic reviews of randomized controlled trials (second update, January 2006) J. Hum. Nutr. Diet., 19, 401-419.

Nabulsi, A.A., Folsom, A.R., White, A., Patsch, W., Heiss, G., Wu, K.K. \& Szklo, M. (1993) Association of hormonereplacement therapy with various cardiovascular risk factors in postmenopausal women. The atherosclerosis risk in communities study investigators. N. Engl. J. Med., 328, 1069-1075.

Nwanguma, B.C., Achebe, A.C., Ezeanyika, L.U.S. \& Eze, L.C. (1999) Toxicity of oxidized fats II: Tissue levels of lipid peroxides in rats fed a thermally oxidized corn oil diet. Food Chem. Toxicol., 37, 413-416.

Osterud, B. \& Bjorklid, E. (2003) Role of monocytes in atherogenesis. Physiol. Rev., 83, 1069-1112.

Owu, D.U., Osim, E.E. \& Ebong, P.E. (1998) Serum liver enzymes profile of Wistar rats following chronic consumption of fresh or oxidized palm oil diets. Acta Trop., 69 , 65-73.

Plutzky, J. \& Libby, P. (2003) Pathophysiology of atherosclerotic heart disease. In: Atherosclerosis and Heart Disease, 1st ed., edited by A.M. Tonkin. Martin Dunitz, London, UK, pp. 1-12.

Rossouw, J.E. (2002) Hormones, genetic factors and gender differences in cardiovascular disease. Cardiovasc. Res., 53 , 550-557.

Rueda-Clausen, C.F., Silva, F.A., Lindarte, M.A., Villa-Roel, C., Gomez, E., Gutierrez, R., Cure-Cure, C. \& LópezJaramillo, P. (2007) Olive, soybean and palm oils intake have a similar acute detrimental effect over the endotelial function in healthy young subjects. Nutr. Metab. Cardiovas. Dis., 17, 50-57.

Staprans, I., Rapp, J.H., Pan, X., Hardman, D.A. \& Feingold, K.R. (1996) Oxidized lipids in the diet accelerate the development of fatty streaks in cholesterol-fed rabbits. Arterioscl. Throm. Vasc. Biol., 16, 533-538.

Su, S., Huang, L., Pai, L., Liu, H. \& Chang, K. (2005) Homocysteine at pathophysiologic concentrations activates human monocyte and induces cytokine expression and inhibits macrophage migration inhibitory factor expression. Nutrition, 21, 994-1002. 